\title{
Efficacy and safety of intermittent versus continuous dose apatinib plus docetaxel as second-line therapy in patients with advanced gastric cancer or gastroesophageal junction adenocarcinoma: a randomized controlled study
}

\author{
Ying Yan ${ }^{1}$, Huimin $\mathrm{Li}^{1}$, Shusheng Wu ${ }^{1}$, Gang Wang ${ }^{1}$, Huiqin Luo ${ }^{1}$, Jiayu Niu ${ }^{1}$, Lulu Cao ${ }^{1}$, Xiaoxiu Hu ${ }^{1}$, \\ Huijun $\mathrm{Xu}^{1}$, Wei Jia ${ }^{1}$, Yubei Sun ${ }^{1}$, Yiwei Yao ${ }^{1}$, Wenju Chen ${ }^{1}$, Lihong Ke ${ }^{1}$, Bing Hu${ }^{1}$, Chushu Ji ${ }^{1}$, \\ Yancai Sun ${ }^{2}$, Jian Chen ${ }^{1}$, Mengge $\mathrm{Li}^{1}$, Yifu $\mathrm{He}^{1}$ \\ ${ }^{1}$ Department of Medical Oncology, The First Affiliated Hospital of USTC, Division of Life Sciences and Medicine, University of Science and \\ Technology of China, Hefei, China; ${ }^{2}$ Department of Pharmacy, The First Affiliated Hospital of USTC, Division of Life Sciences and Medicine, \\ University of Science and Technology of China, Hefei, China \\ Contributions: (I) Conception and design: Y He, B Hu; (II) Administrative support: None; (III) Provision of study materials or patients: All authors; \\ (IV) Collection and assembly of data: Y Yan, H Li, S Wu, H Luo, J Niu, L Cao, X Hu, H Xu, W Jia, W Chen, L Ke, Y Sun, J Chen, M Li, Y He; \\ (V) Data analysis and interpretation: All authors; (VI) Manuscript writing: All authors; (VII) Final approval of manuscript: All authors. \\ Correspondence to: Yifu He, MD, Prof. Department of Medical Oncology, The First Affiliated Hospital of USTC, Division of Life Sciences and \\ Medicine, University of Science and Technology of China, 107 Huanhu East Road, Shushan District, Hefei, China. Email: yifuhe@fsyy.ustc.edu.cn.
}

\begin{abstract}
Background: Previous studies of the second-line treatment for advanced gastric cancer or gastroesophageal junction adenocarcinoma (GC/GEJAC) had reported that apatinib combined with chemotherapy improved the treatment outcomes. However, the benefits were sometimes limited due to the tolerance of continuous dose regimen. This randomized controlled study aimed to investigate the efficacy and safety of intermittent or continuous dose apatinib plus docetaxel as a second-line therapy in patients with advanced GC/GEJAC.

Methods: Advanced GC/GEJAC patients who failed first-line chemotherapy were recruited (enrollment time: from September 15, 2017 to July 21, 2019), and randomly assigned to either the intermittent dose group (IG group) or the continuous dose group (CG group) (1:1 ratio) using the block randomization method. In the IG group, patients received apatinib $500 \mathrm{mg} / \mathrm{d}$ for 5 consecutive days then held for 2 days plus docetaxel $60 \mathrm{mg} / \mathrm{m}^{2} \mathrm{q} 3 \mathrm{w}$, in a 3-week cycle. In the CG group, patients received apatinib $500 \mathrm{mg}$ daily plus docetaxel $60 \mathrm{mg} / \mathrm{m}^{2} \mathrm{q} 3 \mathrm{w}$, in a 3-week cycle. The progression free survival (PFS) was evaluated every two cycles and follow-ups were performed monthly. The primary endpoint was PFS, and the secondary endpoints were objective response rate (ORR), disease control rate (DCR), overall survival (OS), and safety.

Results: In total, 76 eligible patients were enrolled and randomly assigned (1:1 ratio). The IG group exhibited similar PFS compared to the CG group [median PFS: 3.88 (95\% CI: 1.72-6.03) months vs. 3.98 (95\% CI: 1.06-6.90) months, $\mathrm{P}=0.546$ ] and OS [median OS: 9.00 (95\% CI: 5.31-12.70) months vs. 9.40 (95\% CI: 5.20-13.59) months, $\mathrm{P}=0.310]$. ORR (21.1\% vs. $18.4 \%, \mathrm{P}=0.773)$ and $\mathrm{DCR}(60.5 \%$ vs. $60.5 \%, \mathrm{P}=1.000)$ were of not statistically different between the IG and CG groups. As for safety, the IG group exhibited less frequent hypoproteinemia $(31.6 \%$ vs. $55.3 \%, \mathrm{P}=0.037)$ and lactate dehydrogenase increased $(18.4 \%$ vs. $44.7 \%, \mathrm{P}=0.014$ ), while no differences in other adverse events were observed between the two groups.

Conclusions: Intermittent dose apatinib plus docetaxel was equally effective and more tolerable than continuous dose apatinib plus docetaxel as a second-line therapy in patients with advanced GC/GEJAC.
\end{abstract}

Trial Registration: ClinicalTrials.gov NCT03334591.

Keywords: Apatinib; docetaxel; intermittent dose; continuous dose; gastric cancer or gastroesophageal junction adenocarcinoma (GC/GEJAC) 
Submitted Dec 17, 2021. Accepted for publication Feb 18, 2022.

doi: $10.21037 / \mathrm{atm}-22-546$

View this article at: https://dx.doi.org/10.21037/atm-22-546

\section{Introduction}

Gastric cancer (GC), as one of the most common and deadly cancers worldwide, accounts for 572,000 newly diagnosed cases and 311,000 cancer-related deaths annually, and has a high incidence and prevalence in East Asia, Eastern Europe, and South America (1). The etiology of GC is still unknown; however, Helicobacter pylori infection, environmental factors, and inheritance are considered to be important triggers $(2,3)$. Although awareness and early screening programs have been improved to some extent, more than $80 \%$ of GC cases are initially diagnosed at an advanced disease stage, leading to a worse prognosis and a 5 -year survival rate of less than $20 \%(4,5)$. Therefore, to improve the prognosis of patients, exploring treatment options is essential.

Recently, anti-angiogenic therapy has been introduced as an important treatment option for several cancers, including GC (6,7). Apatinib, as a recently developed smallmolecule vascular endothelial growth factor receptor 2 (VEGFR-2) inhibitor that inhibits endothelial cell viability and mobility, thereby blocking tumor angiogenesis, has been proposed to treat GC or gastroesophageal junction adenocarcinoma (GC/GEJAC), and has exhibited good efficacy with acceptable tolerance $(8,9)$. A randomized, placebo-controlled, parallel-arm, phase II trial observed that apatinib prolongs progression-free survival (PFS) and overall survival (OS) in metastatic GC patients who experience treatment failure with at least two chemotherapeutic regimens (10). Another randomized, double-blind, placebo-controlled phase III trial discovered that apatinib significantly improves PFS and OS in advanced GC/GEJAC patients who failed to at least two lines of prior chemotherapy (9). As for the second-line treatment for advanced GC/GEJAC, previous studies have reported that apatinib or ramucirumab combined with chemotherapy improved the treatment outcomes in these patients $(3,11,12)$. However, due to the relatively poor physical conditions and toxicity, the dose of apatinib is often tapered or discontinued during treatment, which limits its benefits to a certain degree. Therefore, better solutions, such as a lower dose strategy that lowers the administered dose each time, were proposed. And this lower dose strategy of apatinib had been applied in treatment of several cancers, for example, advanced non-small cell lung cancer, with good efficacy and tolerable adverse events (13). Although there were experience in continuous lower dose administration of apatinib in the real-world studies (14), no reports examining this lower dose strategy of apatinib, which could be briefly summarized as 5 days administration plus a 2-day gap per week, plus chemotherapy in treating GC/GEJAC as a second-line therapy.

Therefore, this randomized controlled study aims to investigate the efficacy and safety of intermittent or continuous dose apatinib plus docetaxel as a second-line therapy in patients with advanced GC/GEJAC. We present the following article in accordance with the CONSORT reporting checklist (available at https://atm.amegroups. com/article/view/10.21037/atm-22-546/rc).

\section{Methods}

\section{Patients}

In this randomized controlled study, advanced GC/ GEJAC patients who failed first-line chemotherapy were consecutively recruited. The inclusion criteria were as follows: (I) diagnosed as advanced GC/GEJAC; (II) aged $\geq 18$ years; (III) failure of the first-line chemotherapy; (IV) Eastern Cooperative Oncology Group (ECOG) performance status (PS) score of 0-2; (V) patients with at least one measurable lesion; and (VI) those with a life expectancy $>3$ months. The exclusion criteria were as follows: (I) patients that were hypersensitive to medicine composition of apatinib or docetaxel; (II) contraindications to the study drugs, such as active bleeding, ulcers, intestinal perforation, intestinal obstruction, uncontrolled hypertension, within 30 days after major surgery, grade 3-4 cardiac insufficiency (NYHA standard), and severe hepatic and renal insufficiency (grade 4); (III) unable to take oral medicine; (IV) unable to be regularly followed up; (V) complicated with other primary cancers; and (VI) pregnant or breastfeeding females. All procedures performed in this study involving human participants were in accordance with the Declaration of Helsinki (as revised in 2013). The study was approved by ethics board of The First Affiliated Hospital of USTC (No. 2017-07) and informed consent was taken from all the patients. 


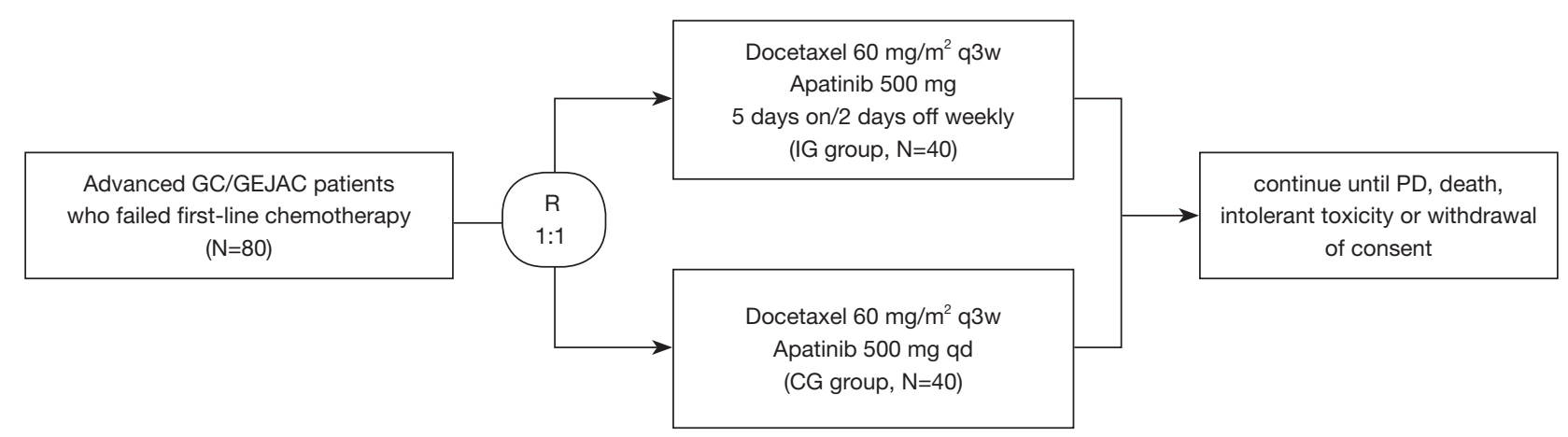

Figure 1 Study design flow chart. GC, gastric cancer; GEJAC, gastroesophageal junction adenocarcinoma; IG group, intermittent dose group; CG group, continuous dose group; PD, progressive disease.

\section{Randomization and procedures}

This study planned to enroll 80 patients and all enrolled patients were randomized to two groups by the ratio of $1: 1$ using block randomization method with block size 4 . SAS 9.4 was used to generate the randomization list and MS EXCEL was used to conduct the randomization process.

After the eligibility of patients was confirmed, the patients were randomly assigned to either the intermittent dose group (IG group) or the continuous dose group (CG group). The trial oversight, database management, and quality assurance were performed at the Department of Medical Oncology, The First Affiliated Hospital of USTC, Division of Life Sciences and Medicine, University of Science and Technology of China. In the IG group, patients received an intermittent dose of apatinib for 5 consecutive days, then held for 2 days, combined with docetaxel $60 \mathrm{mg} / \mathrm{m}^{2}$ ivgtt $\mathrm{q} 3 \mathrm{w}$; both apatinib and docetaxel were continued until the occurrence of progressive disease (PD), death, intolerant toxicity or withdrawal of consent. In the CG group, patients received a continuous dose of apatinib $500 \mathrm{mg} / \mathrm{d}$ p.o. daily combined with docetaxel $60 \mathrm{mg} / \mathrm{m}^{2}$ ivgtt $\mathrm{q} 3 \mathrm{w}$; both apatinib and docetaxel were continued until $\mathrm{PD}$, death, intolerant toxicity or withdrawal of consent (Figure 1). Safety assessments included blood pressure, ECOG performance status, blood pressure, laboratory examinations, and electrocardiogram (every 3 weeks), up to 30 days after treatment discontinuation.

\section{Outcomes assessment}

The primary outcome was PFS (the time from randomization to first disease progression, as assessed by central review according to RECIST, version 1.1, or death from any cause).
The secondary outcomes included OS (the time from randomization to death from any cause), objective response rate (ORR), disease control rate (DCR), and adverse events. The PFS and OS were evaluated by monthly follow-up. Tumor response was examined by computed tomography (CT)/magnetic resonance imaging (MRI) according to the Response Evaluation Criteria in Solid Tumors (RECIST) version 1.1 (15). The ORR was calculated as the percentage of patients who achieved complete response (CR) or partial response (PR). The DCR was calculated as the percentage of patients who achieved CR, PR, or stable disease (SD). The adverse events that occurred during the study were all recorded in detail and graded according to the National Cancer Institute (NCI) Common Terminology Criteria for Adverse Events, version 4.03 (16).

\section{Statistical analysis}

This study was a randomized, controlled clinical trial. The primary endpoint was the PFS evaluated by investigator per RECIST v1.1. A sample size of 80 patients ( 40 for each group), to achieve approximately 73 PFS events, would provide $80 \%$ power with an assumed PFS HR of 0.52 and a two-sided $\alpha$ value of 0.05 .

Data were described as count with percentage or median with $95 \%$ confidence interval (CI). Comparison of the categorical variables between the two groups was determined by the Chi-square test, Fisher's exact test, or Wilcoxon rank-sum test (for categorical variables). Survival curves were constructed using the Kaplan-Meier method and determined by the Log-rank test. SPSS 20.0 statistical software (IBM Corp., Armonk, New York, USA) was used for data analysis. $\mathrm{P}<0.05$ indicated statistical significance. 


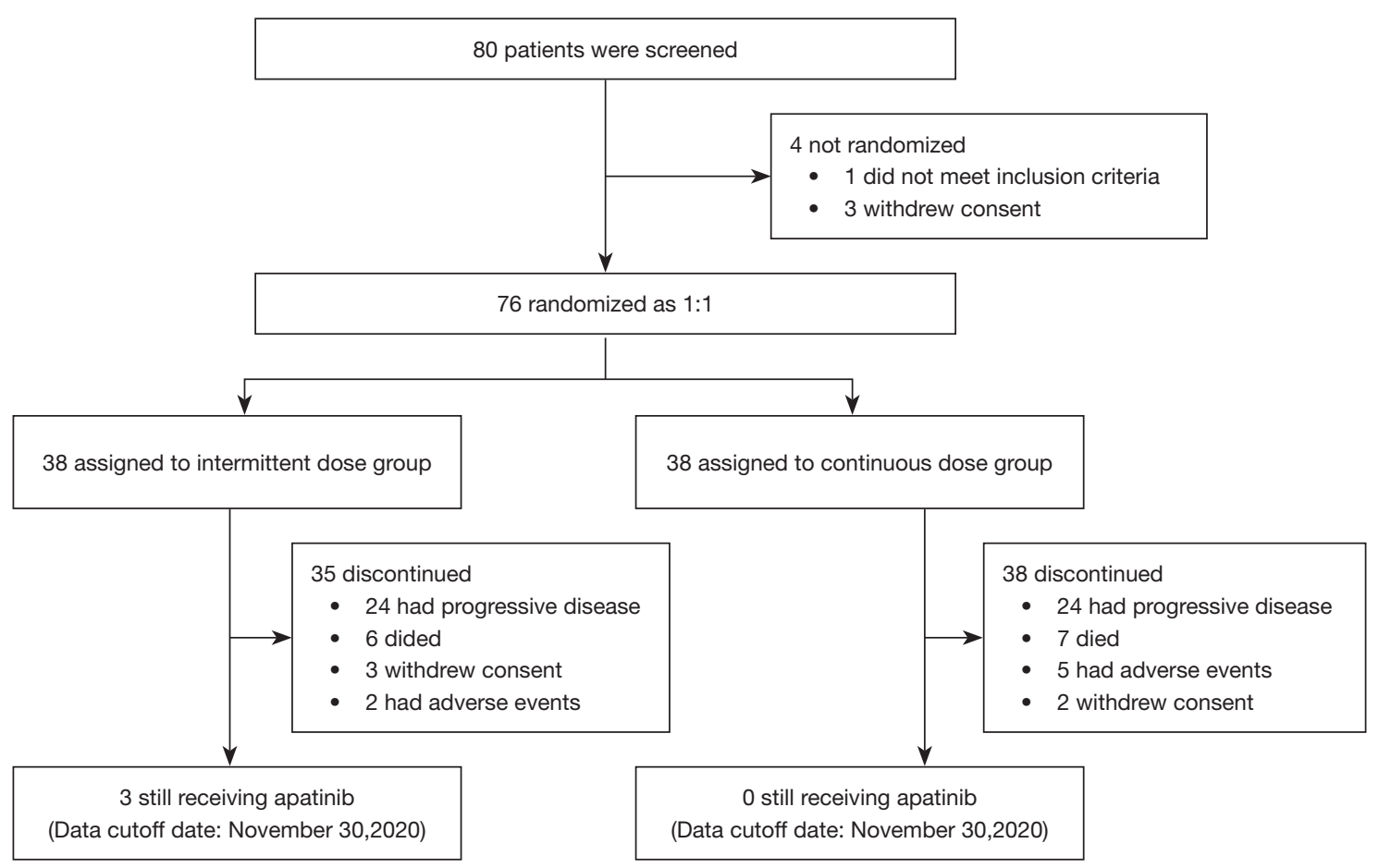

Figure 2 Study flow chart.

\section{Results}

From September 2017 to August 2019, a total of 80 patients were screened, among which four cases were excluded, and the remaining 76 eligible patients were randomly assigned into either the IG group $(n=38)$ or CG group $(n=38)$ in 1:1 ratio (Figure 2). And follow-ups were performed monthly until November 30, 2020. In the IG group, 35 patients discontinued treatment due to PD $(n=24)$, death $(n=6)$, withdrawal of consent $(n=3)$, or adverse events $(n=2)$, and three patients then still received the treatment until the last follow up. In the CG group, 38 patients discontinued due to PD $(n=24)$, death $(n=7)$, adverse events $(n=5)$, or withdrawal of consent $(\mathrm{n}=2)$, and no patients continued to receive the treatment until the last follow up. The detailed baseline features of patients between the IG group and CG group are displayed in Table 1. No difference of age, sex, ECOG PS score, primary tumor site, metastatic lesion number, signet-ring cell carcinoma, history of surgery, or previous chemotherapy regimens was observed between the two groups $(\mathrm{P}>0.05)$.

Moreover, the mean, median, and range of the total actual dose of apatinib were 42,901.3, 32,000.0, and
$12,000.0-177,000.0 \mathrm{mg}$ in the IG group, respectively; and $49,980.3,38,625.0$, and $7,000.0-217,500.0 \mathrm{mg}$ in the CG group, respectively.

\section{Primary outcome}

The IG group exhibited a median PFS of 3.88 months (95\% CI: 1.72-6.03 months), which was of no different to that of the CG group (median PFS: 3.98 months, 95\% CI: 1.066.90 months, $\mathrm{P}=0.546$, Figure 3).

\section{Secondary outcomes}

The IG group achieved 21.1\% PR, 39.5\% SD, and 31.6\% $\mathrm{PD}$, resulting in an ORR of $21.1 \%$ and a DCR of $60.5 \%$. As for the CG group, $18.4 \%$ PR, $42.1 \%$ SD, and $34.2 \%$ PD were achieved, resulting in an ORR of $18.4 \%$ and a DCR of $60.5 \%$. Further comparison showed that there was no difference in the treatment response between the two groups ( $\mathrm{P}>0.05$, Table 2). Furthermore, the OS also showed no difference between the IG and CG groups [median OS: 9.00 (95\% CI: 5.31-12.70) months vs. 9.40 (95\% CI: 5.20 13.59) months, $\mathrm{P}=0.310$, Figure 4]. 
Table 1 Baseline characteristics of the included patients

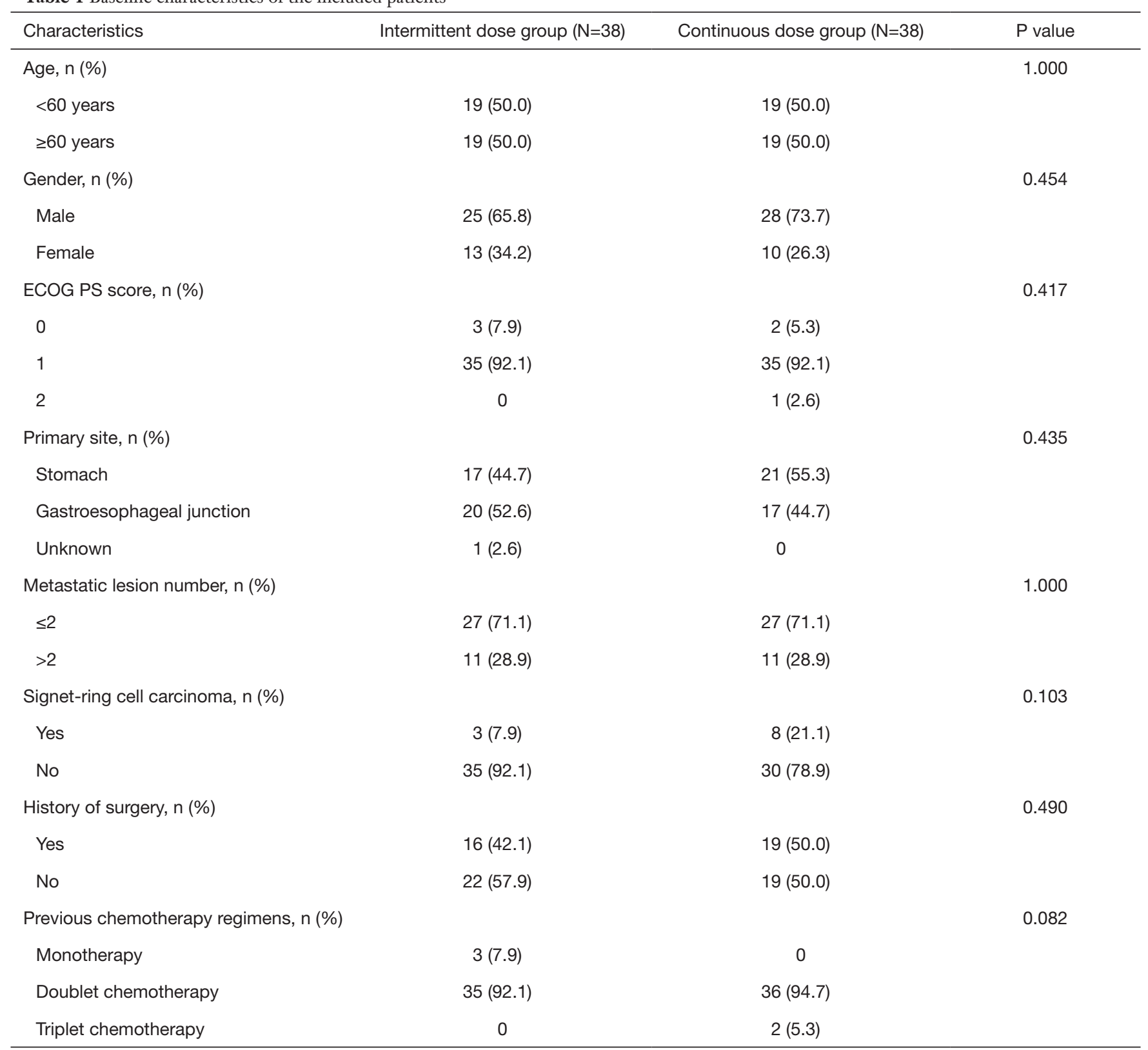

ECOG PS, Eastern Cooperative Oncology Group Performance Status.

\section{Adverse events}

A total of $94.7 \%$ of patients in the IG group and $92.1 \%$ in the CG group presented with adverse events; meanwhile, $36.8 \%$ patients in IG group and $39.5 \%$ patients in CG group suffered from grade $\geq 3$ adverse events.

Notably, hypoproteinemia ( $31.6 \%$ vs. $55.3 \%, \mathrm{P}=0.037$ ) and lactate dehydrogenase increased $(18.4 \%$ vs. $44.7 \%$, $\mathrm{P}=0.014)$ were less frequent in the IG group compared with the CG group (Table 3). In addition, hypertension ( $55.3 \%$ vs. $65.8 \%)$, anemia ( $55.3 \%$ vs. $63.2 \%)$, proteinuria (26.3\% vs. $31.6 \%)$, and hand-foot syndrome (21.1\% vs. $26.3 \%)$, as the common adverse events, were numerically lower in the IG group compared with CG group, although there was no statistical significance. Furthermore, seven cases experienced dose reduction to $250 \mathrm{mg}$ in the IG group, while 13 cases received this dose reduction in the 


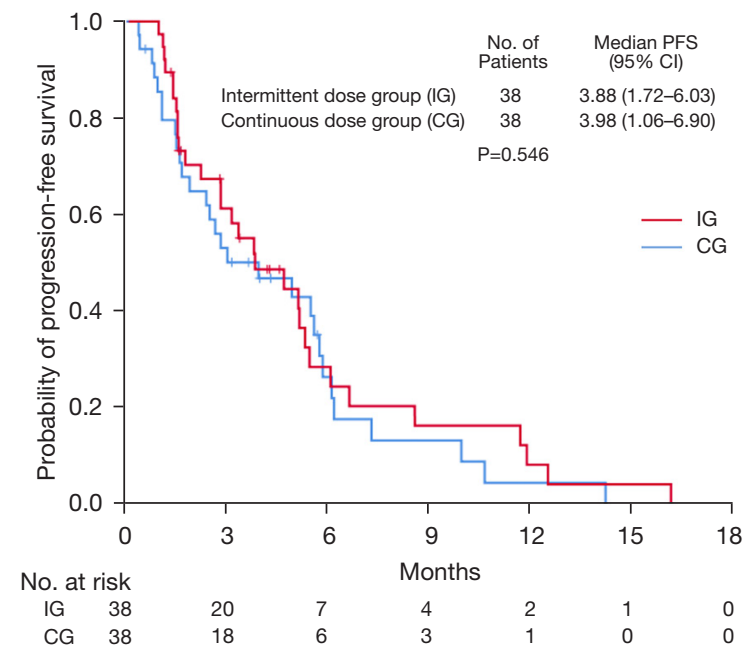

Figure 3 Progression-free survival analysis.

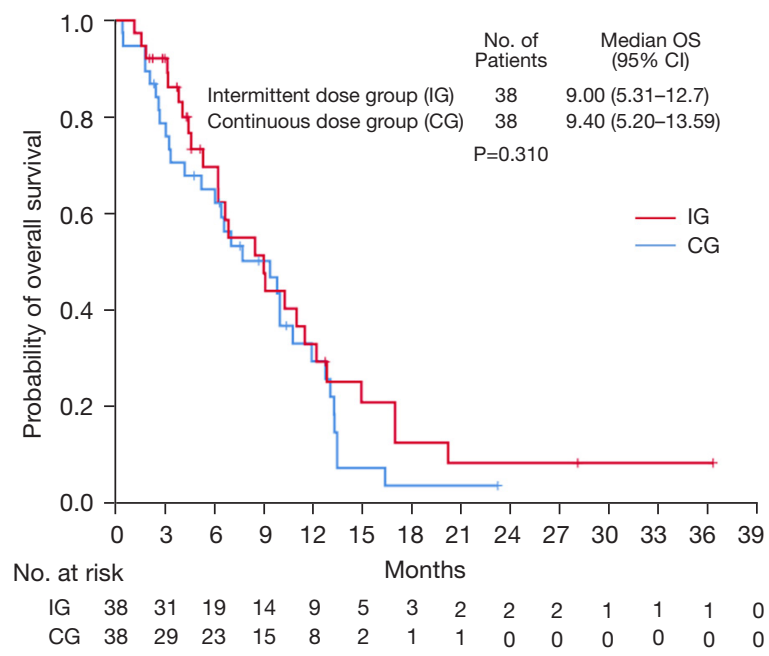

Figure 4 Overall survival analysis.

Table 2 Tumor responses

\begin{tabular}{lccc}
\hline Best overall response & Intermittent dose group $(\mathrm{N}=38)$ & Continuous dose group $(\mathrm{N}=38)$ & $\mathrm{P}$ value \\
\hline PR & $8(21.1)$ & $7(18.4)$ & 0.773 \\
SD & $15(39.5)$ & $16(42.1)$ & 0.815 \\
PD & $12(31.6)$ & $13(34.2)$ & 0.807 \\
UK & $3(7.9)$ & $2(5.3)$ & 1.000 \\
ORR & $8(21.1)$ & $7(18.4)$ & 0.773 \\
DCR & $23(60.5)$ & $23(60.5)$ & 1.000 \\
\hline
\end{tabular}

Data were presented as n (\%). PR, partial response; SD, stable disease; PD, progressive disease; UK, unknown; ORR, objective response rate; $\mathrm{DCR}$, disease control rate.

\section{CG group.}

\section{Discussion}

Several interesting findings were observed in the present study: (I) intermittent dose apatinib plus docetaxel achieved a similar treatment response, PFS, and OS compared to continuous dose apatinib plus docetaxel as a second-line therapy in patients with advanced GC/GEJAC; (II) less adverse events occurred in the IG group compared to the CG group in terms of increased hypoproteinemia lactate dehydrogenase, suggesting that an intermittent dose of apatinib might be safer than a continuous dose of apatinib in these patients.

Advanced GC/GEJAC is dismal regarding its quick progression and poor prognosis, it is now recommended that platinum and fluorouracil-based chemotherapy be used as first-line treatment for these patients, which improves the outcomes to some extent (17). However, there remains a proportion of patients who are refractory or fail first-line chemotherapy. For these patients, paclitaxel, docetaxel, or irinotecan is encouraged; however, recent options consider that monotherapy of paclitaxel, docetaxel, or irinotecan provides restricted benefits in these patients $(3,17)$. In order to resolve this issue, great efforts never stop. Notably, increasing studies have reported that the addition of antiangiogenic agents to chemotherapy as a second-line therapy would further facilitate the prognosis of advanced GC/ $\operatorname{GEJAC}(11,12,18)$.

Since becoming commercially available, apatinib has been commonly used to treat GC/GEJAC under various disease conditions (19-22). In terms of second-line therapy 
Table 3 Adverse events

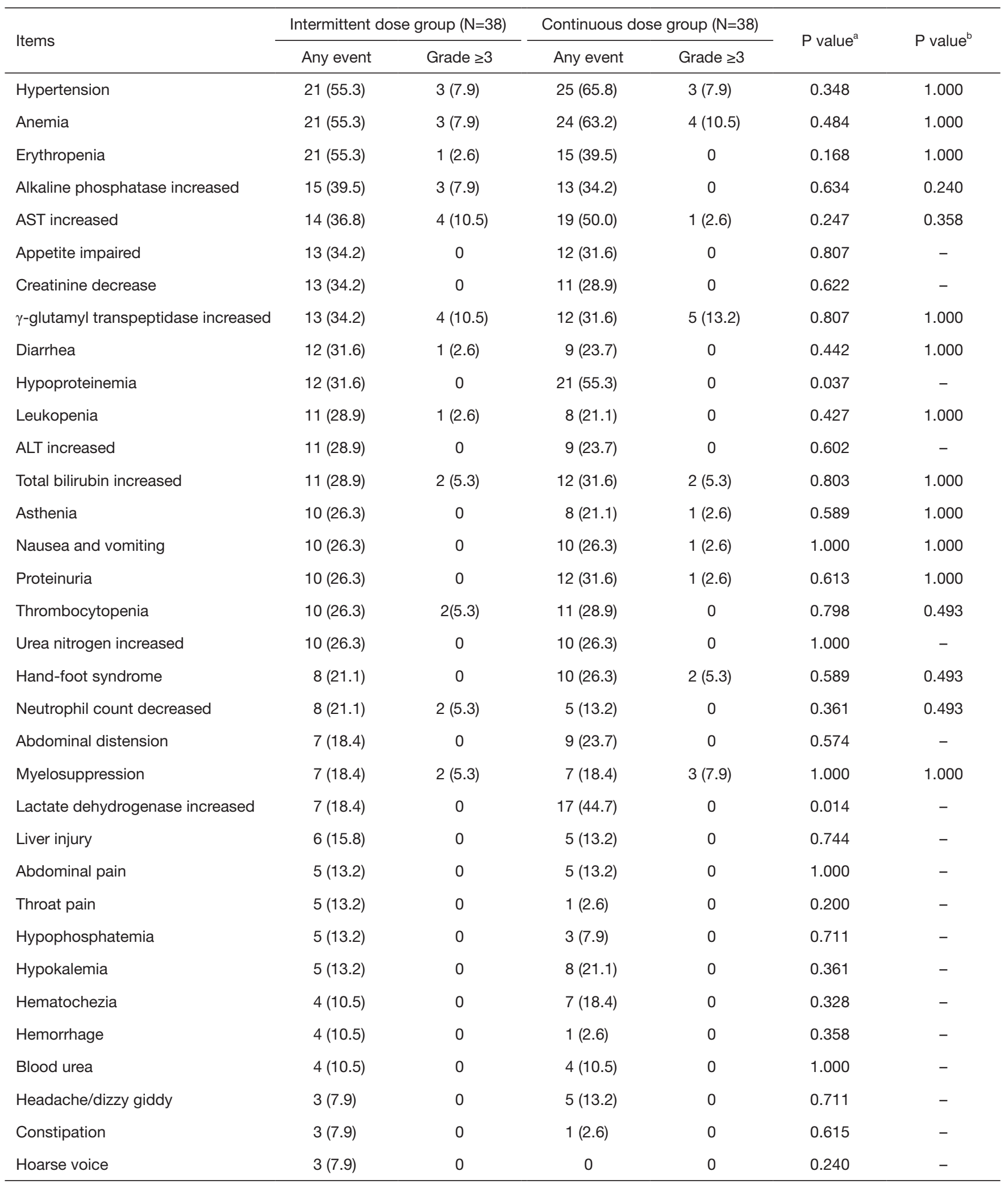

Table 3 (continued) 
Table 3 (continued)

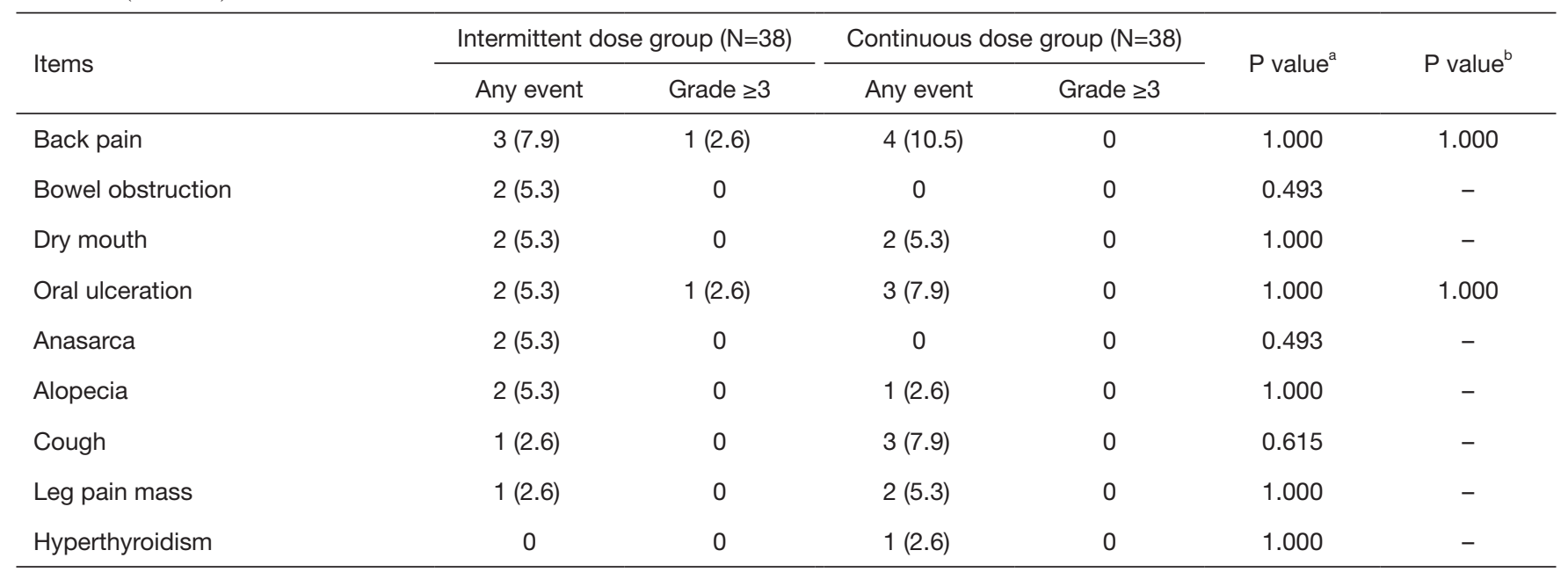

Data were presented as $\mathrm{n}(\%) .{ }^{\mathrm{a}}$, comparison of any event between the intermittent and continuous dose groups; ${ }^{\mathrm{b}}$, comparison of Grade $\geq 3$ between the intermittent and continuous dose groups. AST, aspartate aminotransferase; ALT, alanine aminotransferase.

for these patients, a previous retrospective cohort study discovered that apatinib plus chemotherapy improves the DCR and PFS compared with chemotherapy alone as second- or later-line therapy in GC/GEJAC, with good tolerance (12). Another prospective cohort study found that the addition of apatinib to chemotherapy promotes DCR and PFS compared with chemotherapy alone, and independently correlates with less disease progression after multivariate adjustment as a second- or later-line therapy in GC/GEJAC (23). Furthermore, a randomized controlled study showed that apatinib plus second-line chemotherapy achieved a better DCR, PFS, and OS, as well as fewer adverse events compared to chemotherapy alone in patients with advanced GEJAC (24). In our study, the numerical ORR, DCR, and PFS in both groups were in line with previous studies, while the OS was numerically longer (median OS of 9.0 months in the IG group and 9.4 months in the CG group) compared with that in previous studies that used chemotherapy alone [median OS of 5.2 months in the COUGAR-02 study (docetaxel), 7.4 months in the RAINBOW study (paclitaxel), and 8.3 months in the Keynote-061 study (paclitaxel)] $(11,25,26)$. The possible explanations for this are as follows: (I) apatinib synergizes with chemotherapy drug as previously reported by two experiments $(27,28)$, indirectly resulting in better treatment outcomes in the studied patients; and (II) apart from the synergistic effect, apatinib could directly repress angiogenesis, leading to a more satisfactory prognosis in the studied patients.
Despite the acceptable efficacy of apatinib plus chemotherapy in treating GC/GEJAC as a second-line therapy, the dose is commonly tapered or even stopped during the long-term treatment period, which mainly results from relatively poor physical conditions and toxicity. Therefore, it is essential to identify better solutions to this issue, such as a lower dose strategy that lowers the administered dose each time or reduces the amount of times that it is administered. Low-dose apatinib has been applied in the treatment of several cancers with good efficacy and tolerable adverse events, such as advanced nonsmall cell lung cancer, pulmonary, hepatic metastasis of nasopharyngeal carcinoma, etc. $(13,29)$. In terms of GC/ GEJAC, only two single-arm observational studies have revealed that low-dose apatinib is effective and tolerated for advanced GC patients $(14,30)$. However, there are no reports examining the lower dose strategy of apatinib plus chemotherapy in treating GC/GEJAC as a secondline therapy. Encouragingly, our present study observed that intermittent dose apatinib plus docetaxel achieved a similar treatment response, PFS, and OS compared to continuous dose apatinib plus docetaxel as a second-line therapy in patients with advanced GC/GEJAC, which might be attributed to the fact that the 2-day dose gap of apatinib does not affect the long-term synergistic effect to chemotherapy, and thus, the intermittent dose apatinib plus docetaxel achieves an acceptable efficacy.

Safety data were also collected in detail in the present study, which was more comprehensive compared with 
previous studies. We found that less adverse events occurred with intermittent dose apatinib plus docetaxel compared to continuous dose apatinib plus docetaxel, especially in terms of hypoproteinemia $(31.6 \%$ vs. $55.3 \%)$ and increased lactate dehydrogenase (18.4\% vs. 44.7\%). Furthermore, hypertension (55.3\% vs. $65.8 \%)$, anemia (55.3\% vs. $63.2 \%)$, proteinuria $(26.3 \%$ vs. $31.6 \%)$ and hand-foot syndrome ( $21.1 \%$ vs. $26.3 \%)$, as the commonly occurring adverse events, were also numerically lower in the intermittent dose apatinib plus docetaxel group compared with continuous dose apatinib plus docetaxel group. Lastly, intermittent dose apatinib plus docetaxel also achieved a lower apatinib dose reduction rate compared with continuous dose apatinib plus docetaxel. The above results all suggested that intermittent dose apatinib was safer compared with continuous dose apatinib in these patients. This might be due to the fact that intermittent dose apatinib decreases accumulative druginduced toxicity, thus leading to a relatively more tolerable safety profile.

Several limitations existed in this present study that should be noted. Firstly, the sample size of this study was relatively small, which might have resulted in selection bias. Secondly, intermittent dose apatinib (5 days administration plus a 2-day gap per week) was administered using a kind of lower-dose strategy. Therefore, the direct dose reduction strategy of apatinib plus chemotherapy in treating the studied patients needs to be explored in the future.

In conclusion, intermittent dose apatinib plus docetaxel is equally effective and more tolerable than continuous dose apatinib plus docetaxel as a second-line therapy in patients with advanced GC/GEJAC.

\section{Acknowledgments}

We are grateful to all patients, their families, and the site investigators who participated in the study. Part of the Apatinib was contributed by Hengrui Medicine Co., Ltd. (Lianyungang, China).

Funding: This study was supported by Hefei Key Common Technology Research and Major Scientific and Technological Achievement Project (No. 2021YL005), Natural Science Foundation of Anhui Province (No. 1808085MH234) and Anhui Province Key Research and Development Program Project (No. 202104j07020044).

\section{Footnote}

Reporting Checklist: The authors have completed the
CONSORT reporting checklist. Available at https://atm. amegroups.com/article/view/10.21037/atm-22-546/rc

Trial Protocol: Available at https://atm.amegroups.com/ article/view/10.21037/atm-22-546/tp

Data Sharing Statement: Available at https://atm.amegroups. com/article/view/10.21037/atm-22-546/dss

Conflicts of Interest: All authors have completed the ICMJE uniform disclosure form (available at https://atm.amegroups. com/article/view/10.21037/atm-22-546/coif). All authors report fundings from Hefei Key Common Technology Research and Major Scientific and Technological Achievement Project (2021YL005), Natural Science Foundation of Anhui Province (1808085MH234) and Anhui Province Key Research and Development Program Project (202104j07020044). Part of the Apatinib was contributed by Hengrui Medicine Co., Ltd. (Lianyungang, China). The authors have no other conflicts of interest to declare.

Ethical Statement: The authors are accountable for all aspects of the work in ensuring that questions related to the accuracy or integrity of any part of the work are appropriately investigated and resolved. All procedures performed in this study involving human participants were in accordance with the Declaration of Helsinki (as revised in 2013). The study was approved by ethics board of The First Affiliated Hospital of USTC (No. 2017-07) and informed consent was taken from all the patients.

Open Access Statement: This is an Open Access article distributed in accordance with the Creative Commons Attribution-NonCommercial-NoDerivs 4.0 International License (CC BY-NC-ND 4.0), which permits the noncommercial replication and distribution of the article with the strict proviso that no changes or edits are made and the original work is properly cited (including links to both the formal publication through the relevant DOI and the license). See: https://creativecommons.org/licenses/by-nc-nd/4.0/.

\section{References}

1. Bray F, Ferlay J, Soerjomataram I, et al. Global cancer statistics 2018: GLOBOCAN estimates of incidence and mortality worldwide for 36 cancers in 185 countries. CA Cancer J Clin 2018;68:394-424.

2. Van Cutsem E, Sagaert X, Topal B, et al. Gastric cancer. 
Lancet 2016;388:2654-64.

3. NCCN Clinical Practice Guidelines in Oncology (NCCN Guidelines). Gastric Cancer. Version 2.2020. 2020.

4. Wang W, Sun Z, Deng JY, et al. A novel nomogram individually predicting disease-specific survival after D2 gastrectomy for advanced gastric cancer. Cancer Commun (Lond) 2018;38:23.

5. Zong L, Abe M, Seto Y, et al. The challenge of screening for early gastric cancer in China. Lancet 2016;388:2606.

6. De Vita F, Di Martino N, Fabozzi A, et al. Clinical management of advanced gastric cancer: the role of new molecular drugs. World J Gastroenterol 2014;20:14537-58.

7. Wong H, Yau T. Molecular targeted therapies in advanced gastric cancer: does tumor histology matter? Therap Adv Gastroenterol 2013;6:15-31.

8. Shen B, Jiang H, Wang L, et al. Effectiveness and Safety of Apatinib in Patients with Advanced or Metastatic Adenocarcinoma of Stomach or Gastroesophageal Junction: A Prospective Observation Study. Onco Targets Ther 2020;13:4457-64.

9. Li J, Qin S, Xu J, et al. Randomized, Double-Blind, Placebo-Controlled Phase III Trial of Apatinib in Patients With Chemotherapy-Refractory Advanced or Metastatic Adenocarcinoma of the Stomach or Gastroesophageal Junction. J Clin Oncol 2016;34:1448-54.

10. Li J, Qin S, Xu J, et al. Apatinib for chemotherapyrefractory advanced metastatic gastric cancer: results from a randomized, placebo-controlled, parallel-arm, phase II trial. J Clin Oncol 2013;31:3219-25.

11. Wilke H, Muro K, Van Cutsem E, et al. Ramucirumab plus paclitaxel versus placebo plus paclitaxel in patients with previously treated advanced gastric or gastro-oesophageal junction adenocarcinoma (RAINBOW): a double-blind, randomised phase 3 trial. Lancet Oncol 2014;15:1224-35.

12. Zhang F, Yin Y, Ni T, et al. Treatment effect of apatinib combined chemotherapy as second-line or above therapy in patients with advanced gastric cancer or adenocarcinoma of the gastroesophageal junction. Pharmazie 2020;75:389-94.

13. Zhou T, Wu C, Zhang C, et al. A retrospective study of low-dose apatinib combined with $\mathrm{S}-1$ in patients with advanced non-small cell lung cancer. J Thorac Dis 2019;11:1831-7.

14. Du Y, Cao Q, Jiang C, et al. Effectiveness and safety of low-dose apatinib in advanced gastric cancer: A real-world study. Cancer Med 2020;9:5008-14.

15. Eisenhauer EA, Therasse P, Bogaerts J, et al. New response evaluation criteria in solid tumours: revised RECIST guideline (version 1.1). Eur J Cancer 2009;45:228-47.

16. Common Terminology Criteria for Adverse Events (CTCAE) Version 4.03. 2010. Available online: https:// ctep.cancer.gov/protocolDevelopment/electronic_ applications/docs/CTCAE_4.03.xlsx

17. Wang FH, Shen L, Li J, et al. The Chinese Society of Clinical Oncology (CSCO): clinical guidelines for the diagnosis and treatment of gastric cancer. Cancer Commun (Lond) 2019;39:10.

18. Chen LT, Oh DY, Ryu MH, et al. Anti-angiogenic Therapy in Patients with Advanced Gastric and Gastroesophageal Junction Cancer: A Systematic Review. Cancer Res Treat 2017;49:851-68.

19. Shao F, Zhang H, Yang X, et al. Adverse events and management of apatinib in patients with advanced or metastatic cancers: A review. Neoplasma 2020;67:715-23.

20. Zhang C, Yu GM, Zhang M, et al. S-1 plus apatinib as first-line palliative treatment for stage IVB gastroesophageal junction adenocarcinoma: A case report and review of the literature. Medicine (Baltimore) 2020;99:e18691.

21. Cheng H, Sun A, Guo Q, et al. Efficacy and safety of apatinib combined with chemotherapy for the treatment of advanced gastric cancer in the Chinese population: a systematic review and meta-analysis. Drug Des Devel Ther 2018;12:2173-83.

22. Peng Z, Wei J, Wang F, et al. Camrelizumab Combined with Chemotherapy Followed by Camrelizumab plus Apatinib as First-line Therapy for Advanced Gastric or Gastroesophageal Junction Adenocarcinoma. Clin Cancer Res 2021;27:3069-78.

23. Guo Y, Tang J, Huang XE, et al. Efficacy and toxicity of apatinib combined with or without chemotherapy for patients with advanced or metastatic chemotherapyrefractory gastric adenocarcinoma: A prospective clinical study. Medicine (Baltimore) 2019;98:e13908.

24. Lu B, Lu C, Sun Z, et al. Combination of apatinib mesylate and second-line chemotherapy for treating gastroesophageal junction adenocarcinoma. J Int Med Res 2019;47:2207-14.

25. Ford HE, Marshall A, Bridgewater JA, et al. Docetaxel versus active symptom control for refractory oesophagogastric adenocarcinoma (COUGAR-02): an open-label, phase 3 randomised controlled trial. Lancet Oncol 2014;15:78-86.

26. Shitara K, Özgüroğlu M, Bang YJ, et al. Pembrolizumab versus paclitaxel for previously treated, advanced gastric or gastro-oesophageal junction cancer (KEYNOTE-061): a 
randomised, open-label, controlled, phase 3 trial. Lancet 2018;392:123-33.

27. Xu Z, Hu C, Chen S, et al. Apatinib enhances chemosensitivity of gastric cancer to paclitaxel and 5-fluorouracil. Cancer Manag Res 2019;11:4905-15.

28. Feng J, Qin S. The synergistic effects of Apatinib combined with cytotoxic chemotherapeutic agents on gastric cancer cells and in a fluorescence imaging gastric cancer xenograft model. Onco Targets Ther 2018;11:3047-57.

29. Zhou L, Lin J, Wu G, et al. Safety and Feasibility of Low-

Cite this article as: Yan Y, Li H, Wu S, Wang G, Luo H, Niu J, Cao L, Hu X, Xu H, Jia W, Sun Y, Yao Y, Chen W, Ke L, Hu B, Ji C, Sun Y, Chen J, Li M, He Y. Efficacy and safety of intermittent versus continuous dose apatinib plus docetaxel as second-line therapy in patients with advanced gastric cancer or gastroesophageal junction adenocarcinoma: a randomized controlled study. Ann Transl Med 2022;10(4):205. doi: 10.21037/atm-22-546
Dose Apatinib Combined with S-1 as the Second-Line Therapy or Beyond in Chinese Patients with Pulmonary and Hepatic Metastasis of Nasopharyngeal Carcinoma. Drug Des Devel Ther 2020;14:1257-62.

30. Chen J, Wang J, Miao Q. Careful dose modification of apatinib as third or further-line treatment in advanced gastric cancer patients with poor performance status. Medicine (Baltimore) 2019;98:e17890.

(English Language Editor: A. Kassem) 